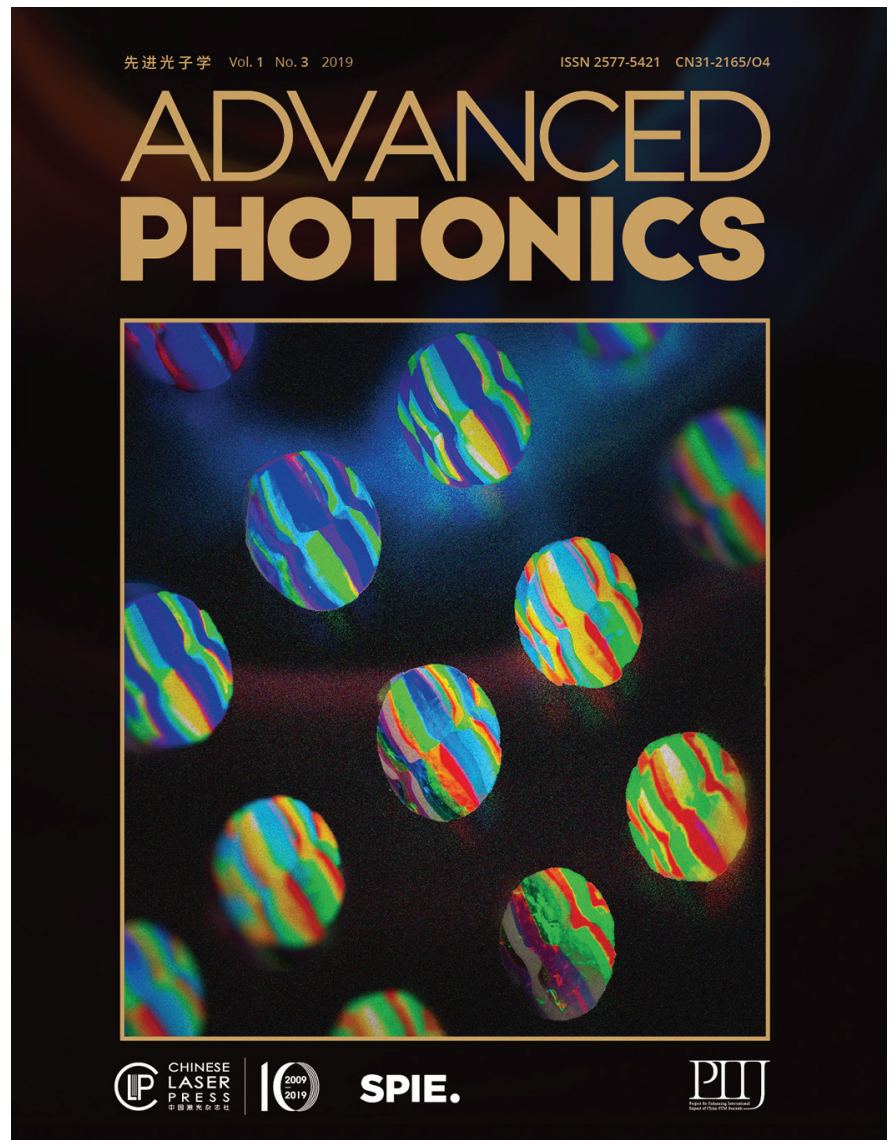

\title{
About the cover: Advanced Photonics Volume 1, Issue 3
}

The image on the cover for Advanced Photonics Volume 1, Issue 3, depicts nanostructured optical elements consisting of resonant periodic metasurfaces made from silicon nanodiscs. These optical elements, by changing their lateral geometrical parameters, allow control of the phase of transmitted light, which enables three-dimensional imaging with a single camera. Colors in the photograph are generated by the different periodicities used, which diffract light differently.
The image is an artistic rendering of the role of resonant periodic metasurfaces in producing complex optical functionalities, based on the research presented by Chunqi Jin et al., in their article, "Dielectric metasurfaces for distance measurements and three-dimensional imaging," Advanced Photonics, 1(3), 036001, doi: 10.1117/1.AP.1.3.036001. 